\title{
Questions concerning speciation
}

\section{from Ernst Mayr}

THE pattern of evolution in the mollusc lineages reported by Williamson seems to be in excellent agreement with the theory of punctuated equilibria, which is based on the well-known paleontological observation that new species usually enter the fossil record abruptly (in geological time) and postulates that they persist subsequently with little significant change until extinction. The rapid change under the stress conditions of an evaporating Lake Turkana is, as Williamson points out, less of a puzzle than the millions of years of stasis.

Williamson refers to my observation that many currently living species show phenotypic uniformity even though their range extends over areas of considerable ecological and climatic diversity. This observation strengthens the thesis that it is not stabilizing selection of a set of essentially additive genes that is responsible for the stasis but rather the strength of the "internal balance", (Mather), "genetic homeostasis" (Lerner), "cohesion of the genotype" (Mayr). These authors postulate that the genotype as a whole is a finely balanced system, in which appropriate feedback mechanisms maintain morphological stability by compensating for whatever genetic changes occur through time at individual loci.

Williamson has presented a plausible explanation of his findings and it might well be the correct one. However, in his short communication, he has not refuted conceivable alternate theories nor has he provided answers to some disturbing questions that might arise in the mind of the reader.

First, Williamson states that the changes in 12 lines of sexually reproducing molluscs are paralleled exactly by events in the lineage of the asexually reproducing species Melanoides tuberculatus. Since an asexual phyletic lineage consists of hundreds, if not many thousands, of independent clones, how does Williamson explain that all of them experience a parallel history of equivalent genetic changes? This concordant phenotypic change of the scores of asexual clones suggests that the changes are not genetic at all, but merely modifications of the phenotype (see also the letter below from A.J. Boucot;

Second, does the variability of the asexual (Melanoides) lineage, consisting of numerous independent clones, differ from those of the sexual lineages?

Third, Williamson states that the new 'species' become suddenly extinct when the

Ernst Mayr is Alexander Agassiz Professor, Emeritus, in the Museum of Comparative Zoology, Harvard University, Cambridge, MA 02138 . widespread parental species reinvade the Turkana basin. There are four sets of possibilities for the behaviour of parental and daughter "species" at the time of the establishment of sympatry: (a) Conditions in the Turkana basin, before the reinvasion by the widespread parental stocks, had deteriorated to such an extent that the new "species" had all become extinct already; (b) They had become reproductively isolated; in that case it should be possible to find them sympatrically in zones of contact; (c) They had not yet developed reproductive isolation and it should be possible to find hybrid populations with greatly increased variability; (d) The observed morphological differences of mother and daughter 'species' had no genetic basis but were merely phenotypic responses to different environmental conditions; in that case a sudden and total change of the phenotype should accompany the replacement of the lake waters. A finegrained analysis of the populations occurring during the crucial period of reversion to standard conditions should permit an answer to these questions.

Finally, one would also like to know more about one set of puzzling speciations. In six of the 13 lineages a second species apparently originated in the lower Koobi Fora Formation (Bellomya unicolor, Cleopatra ferruginea, Melanoides tuberculatus, Caelatura bakeri, Mutela nilotica, and Eupera ferruginea). All six of these daughter species seem to become extinct before the end of the lower Koobi Fora (see Fig. 4). Do these six new daughter species coexist sympatrically with the parent stock without producing intermediates? Does Williamson believe that they originated in some peripheral isolate (a temporarily isolated lagoon?) and were able, after a rise in lake level, to invade the range of the parent stock? Several other questions are obvious, but one can not even begin to answer them until Williamson produces more factual evidence about these six postulated speciation events.

Williamson states that, on the whole, his findings are consistent with my theory of peripatric speciation in peripheral founder populations, with two drastic exceptions: The asexual Melanoides behaves like the sexual lineages, a fact not explained by Mayr's postulate of genetic revolutions, and the size of the shell deposits indicates that the 13 (19) lineages never truly seem to have passed through a narrow populational bottleneck, as demanded by the theory of peripatric speciation. It is impossible to resolve this conflict Williamson himsclf does not try to do it until much better factual evidence for some of the events in the sequence of these mollusc faunas is presented.

\section{Ecophenotypic or genotypic?}

\section{from Arthur J. Boucot}

WILLIAMSON's brief comment (p. 441) that the presumably derivative morphologies, all arrayed in two, well-dated, very discrete horizons and one more diffuse horizon, cannot be considered to be ecophenotypic morphologies of the unchanging, longranging forms - many of which persist to the present - needs discussion. It is essential that in an area where lake waters of widely different composition are known to have occurred in the past, as well as the present, that the possibility of ecophenotypic, as contrasted with a genetic, change be dismissed only when adequate data has accumulated. One would have hoped that Williamson would have provided us with observational data, based on those species that persist to the present, showing that under conditions of varied lake water composition (both concentration and composition) there are no ecophenotypic effects.

If such data are unavailable, Williamson could have cultured at least some of the living species under conditions of salinity and water composition that might have occurred in parts of the Turkana Basin region during the time interval studied. Without such data one must be forgiven for still considering that the possibility of ecophenotypic change is fully as rational as that of speciation. Few neontologists would adhere to the tenet that distinctly different skeletal morphologies within a genus invariably indicate different genotypes. Paleontologists nosmally assign distinctly different morphologies within a genus to different species, lacking evidence for a well documented morphologic gradient of the ecophenotypic type, only because they commonly deal with extinct genera; not because they feel that the procedure is invariably defensible.

Finally, Williamson really should have provided us with a satisfying explanation of why speciational events should have occurred simultaneously, at two distinct

Arthur J. Boucot is in the Department of Geology, Oregon State University, Corvallis, Oregon 97311. 